\title{
INSUFICIENCIA ADRENAL EN EL PACIENTE CRÍTICO
}

Mario Gómez MD F.C.C.M*, Guillermo Pinzón Velásquez MD**

\section{Resumen}

La insuficiencia adrenal (ISA) en la unidad de cuidado intensivo es una situación clínica frecuente en el paciente crítico. Se trata de un espectro clínico que es de relevancia diagnóstica, terapéutica y pronóstica, por las consecuencias y potencial amenaza de la vida cuando no se reconoce a tiempo en los pacientes. En la presente revisión se plantean aspectos fisiopatológicos, diagnósticos y terapéuticos de esta entidad.

insuficiencia adrenal, crisis adrenal aguda, cuidado crítico, terapia con glucocorticoides. ISA, insuficiencia adrenal; GC, glucocorticoides; IAR, insuficiencia adrenal relativa; ACTH, hormona adrenocorticotropica; CRH, hormona corticotropa hipotalámica.

\section{Abstract}

Adrenal insufficiency (AI) is a frequent clinical condition in the critically ill ICU patients. This clinical spectrum bears diagnostic, therapeutic and prognostic relevance due to its consequences and is potentially life threatening if not timely diagnosed. This review describes various pathophysiological, diagnostic and therapeutic aspects of this entity.

adrenal insufficiency, acute adrenal crisis, critical care medicine, glucocorticoid therapy.

\section{Introducción}

La ISA en cuidado crítico es una situación clínica inusual en algunos reportes de la literatura y frecuente para otros, se trata de un espectro clínico de la misma enfermedad que es de relevancia por las consecuencias y potencial amenaza de la vida cuando no se reconoce a tiempo. La ISA puede ser primaria o secundaria como consecuencia de la insuficiencia del

Fecha recibido: octubre 8 de 2007 - Fecha aceptado: noviembre 3 de 2007

* Jefe de la Unidad de Cuidado Intensivo Adultos. Fundación Universitaria de Ciencias de la Salud, Hospital de San José.

** Residente de tercer año de medicina interna. Especialista en seguridad social. Fundación Universitaria de Ciencias de la Salud, Hospital de San José. eje hipotálamo-hipófisis. En otras circunstancias corresponde a cuadros de origen iatrógeno causada por la supresión del eje hipotálamo-hipófisis-glándula adrenal por la administración de glucocorticoides, siendo esta última la forma más común de ISA.

Presentaciones clásicas de la insuficiencia adrenal

La ISA puede dividirse en tres grandes categorías:

1. Insuficiencia adrenal primaria crónica, llamada enfermedad de Addisson, que es el resultado de la destrucción de la corteza adrenal. Es la causa más común, entre $70 \%$ y $80 \%$ de la ISA primaria crónica, seguida de la tuberculosis con un $20 \%$, 
hemorragia, metástasis, infección por citomegalovirus en pacientes con $\mathrm{VIH}$ y tratamiento con ketoconazol.

2. Insuficiencia adrenal crónica secundaria, ocurre cuando la ACTH no está disponible para estimular la corteza adrenal. El uso de terapia con glucocorticoides es la principal causa, aunque también se encuentran tumores hipofisiarios o hipotalámicos, en el contexto de un panhipopituitarismo y menos común la deficiencia de ACTH de origen autoinmune.

3. La crisis adrenal aguda se observa en pacientes con estrés agudo e historia de insuficiencia adrenal crónica sin esquema terapéutico apropiado; también puede presentarse en pacientes con hemorragia adrenal o apoplejía pituitaria.

Ambas condiciones clínicas, tanto la insuficiencia primaria como la secundaria, comparten la deficiencia de GC y en ocasiones también de andrógenos, en especial en la mujer. Los rasgos clínicos comunes a ambas situaciones incluyen hipotensión, fatiga, anorexia, pérdida de peso, náuseas y emesis. La esosinofilia y la anemia normocítica son comunes y a veces puede observarse hipercalcemia. La hipoglicemia suele ocurrir en los niños con ISA primaria y en pacientes con ISA secundaria en el contexto de panhipopituitarismo, cuando la hormona del crecimiento está también disminuida.

La ISA crónica primaria puede acompañarse con otros desórdenes autoinmunes (falla poliglandular), la más común es la enfermedad autoinmune tiroidea (enfermedad de Graves y tiroiditis de Hashimoto), raras veces los pacientes con hipoparatiroidismo se encuentran asociados con la presentación de la enfermedad, pero podrían desarrollar hipocalcemia. Dos rasgos son útiles en la diferenciación de la ISA primaria y secundaria. El primero, la deficiencia de mineralocorticoides se encuentra en la forma primaria y está ausente en la secundaria (la ACTH no juega un rol importante en la regulación de la aldosterona), es por esta razón que la hipercalemia está presente por lo regular en la forma primaria. La hiponatremia se encuentra en ambas, pero en la ISA primaria está asociada con hipovolemia causando elevación de la creatinina y el BUN. La hiponatremia en la ISA secundaria es dilucional por la disminución de la capacidad de excreción de agua y el incremento de los niveles de vasopresina.

El segundo rasgo es la alta concentración de ACTH y otros péptidos derivados de la propiomelanocortina en la ISA primaria y los niveles bajos o normales de estos péptidos en la ISA secundaria. Esta es la razón de la hiperpigmentacion en la forma primaria y la carencia de la misma e inclusive la palidez en la insuficiencia secundaria. La crisis adrenal aguda está caracterizada por hipotensión y choque, fiebre, confusión, náuseas y emesis; si la causa es una hemorragia adrenal podría encontrarse distensión abdominal dolorosa. La apoplejía pituituaria está a menudo asociada con cefalea severa y oftalmoplejía, y las anormalidades de laboratorio incluyen azoemia y eosinofilia.

\section{Fisiopatología}

El eje hipotálamo-hipófisis-adrenal intacto es de vital importancia para los pacientes severamente enfermos. Como respuesta al estrés el hipotálamo secreta $\mathrm{CRH}$, la cual estimula a la hipófisis para producir ACTH y esta a la glándula suprarrenal para liberar cortisol, el GC de mayor relevancia clínica. Los altos niveles de este último ejercen un efecto regulador negativo para la producción de CRH y ACTH.

La glándula adrenal se compone de dos unidades funcionales, la médula que produce hormonas del sistema simpático (epinefrina y norepinefrina) y la corteza que se divide en tres zonas: (1) la glomerulosa localizada en la superficie que se encarga de producir mineralocorticoides (aldosterona), (2) la reticular que produce algunos andrógenos y (3) la fascicular que produce GC (cortisol y cortisona). El cortisol es una hormona esteroidea que circula libre en forma activa entre el 5 y $10 \%$ del total plasmático y también ligada a proteínas (albúmina y globulinas) que es la forma inactiva. 
I. Suprime la función y acumulación de la mayoría de células implicadas en los fenómenos inflamatorios.

2. Estimula la gluconeogénesis por la inhibición de la captación de glucosa en los tejidos periféricos y promueve la producción de ácidos grasos libres del tejido adiposo, además de la producción de aminoácidos a partir de las proteínas.

3. Interviene en el mantenimiento de la contractibilidad miocárdica, el tono vascular y la presión arterial, mediado por el aumento de la acción de la angiotensina II, epinefrina y norepinefrina.

4. Previene la retención excesiva de agua libre durante el estrés por la supresión de la hormona antidiurética, incrementando la tasa de filtración glomerular y alterando la permeabilidad vascular y la distribución del agua corporal total.

El cortisol ingresa a las células de manera pasiva y se liga con su receptor citosólico soluble conocido como receptor de GC tipo II. E1 complejo formado entra al núcleo e interactúa en forma directa con sitios específicos del ADN, iniciando una cadena de trascripción de diferentes genes para las diversas funciones celulares (Tabla 1). El cortisol es metabolizado por el hígado (reducción y conjugación) y por el riñón donde es convertido en metabolitos inactivos de cortisona por acción enzimática. En los pacientes críticos es muy bajo el nivel de globulina fijadora de cortisol, presentándose mayores cantidades del mismo en forma libre. A su vez el metabolismo se encuentra disminuido, así como los receptores de GC con gran afinidad por el mismo.

\section{Eje hipotálamo-hipófisis-adrenal}

La producción y secreción de cortisol son inducidas por la ACTH, que también lo hace para la producción de andrógenos y en menor medida de mineralocorticoides. La secreción de ACTH es estimulada por la $\mathrm{CRH}$ y la vasopresina al igual que otros péptidos como la angiotensina II, serotonina y péptido intestinal vasoactivo. Algunas citoquinas inflamatorias estimulan también la secreción de ACTH: interleucina (IL) 1, IL-2, IL-6 y factor de necrosis tumoral- $\alpha$, mientras otras lo inhiben como el factor de crecimiento- $\alpha$. Muchos otros modulan la secreción de $\mathrm{CRH}$, incluyendo los agonistas adrenérgicos, opioides y citoquinas inflamatorias.
La secreción del eje sigue un patrón de pulso con el ritmo circadiano que es mayor durante la mañana. En situaciones de estrés la concentración de GC en el tejido adrenal no es suficiente para el incremento inicial del cortisol que ocurre en estas situaciones, como tampoco lo es para mantener tasas de secreción normal por unos pocos minutos en ausencia de biosíntesis continua. La tasa de secreción es directamente proporcional a la biosíntesis y cualquier interrupción en la síntesis de corticoides de inmediato resulta en una insuficiencia de los mismos.

\section{Mecanismo de acción de los GC}

Efectos metabólicos: intervienen en el metabolismo de la glucosa estimulando la gluconeogénesis y la glicogenólisis, induciendo la resistencia periférica a la insulina e incrementando la concentración de glucosa plasmática. Actúan favoreciendo la lipólisis e inhiben la captación de glucosa por los adipocitos. Inhiben la síntesis y activación de proteinólisis muscular, liberando aminoácidos que sirven para la gluconeogénesis. Estos activan los osteoclastos, inhiben los osteoblastos y la captación de calcio intestinal e incrementan la excreción urinaria por medio de la disminución en la reabsorción renal.

Efectos inmunológicos y antiinflamatorios: las células inmunes presentan receptores de alta afinidad por los GC. Cuando se administran GC disminuyen los linfocitos circulantes, ya que estos migran hacia los nódulos linfáticos. El efecto opuesto es obser- 
vado con los granulocitos, los cuales se acumulan en la circulación mientras los neutrófilos migran desde de los sitios de inflamación contribuyendo a la disminución de la reacción inflamatoria local. Los macrófagos también reducen su respuesta por la producción de factor inhibidor de macrófagos e inducen apoptosis de los eosinófilos. En la respuesta inmune los GC inhiben la secreción de IL12 por los macrófagos y los monocitos, y su influencia sobre la diferenciación de los linfocitos Th1 a Th2. Esta actividad antiinflamatoria termina con la inhibición de la producción de la ciclooxigenasa y la sintasa inducible del óxido nítrico, que son clave en el proceso inflamatorio.

Efectos cardiovasculares: tienen un rol vital en el mantenimiento del tono vascular, la integridad endotelial, permeabilidad vascular y la distribución del total del agua dentro del compartimiento intravascular. Potencian la acción vasoconstrictora de las catecolaminas y disminuyen la producción de óxido nítrico y otros factores vasodilatadores.

\section{Activación del eje hipotálamo-hipófisis-adrenal durante el estrés}

Sin distinción de la causa física (infección severa, trauma, quemaduras, cirugías) o psicológica, el estrés activa el eje con el incremento de la secreción de ACTH y los niveles sericos de cortisol. Además disminuyen los niveles de proteína fijadora de cortisol con el consecuente aumento de la fracción libre. Las citoquinas también aumentan su afinidad por los GC. Con el estrés, el ritmo circadiano de la secreción diurna de cortisol se pierde y se incrementa su producción que por lo general es proporcional a la severidad de la enfermedad.

Estos efectos son causados por un incremento en la CRH y la ACTH estimulado por las citoquinas proinflamatorias y la reducción en la retroalimentación negativa del cortisol. La hiperglicemia contribuye al aumento de los sustratos energéticos durante el período en que se requiere un incremento en el metabolismo y la disponibilidad de glucosa en las células dependientes de insulina, como ocurre en el sistema nervioso central y las células inflamatorias.

\section{Etiología de la ISA}

Las enfermedades que causan ISA primaria por lo regular destruyen la corteza adrenal en su totalidad, causando una combinación de deficiencia de GC, mineralocorticoides, andrógenos y eventualmente en algunas de ellas insuficiencia de la medula adrenal. Los síntomas clínicos de la ISA primaria son más prominentes que en los casos de ISA secundaria y se pueden iniciar con crisis adrenal aguda.

Las causas de la ISA secundaria son más selectivas en el deterioro de la función GC que la minerolocorticoide, ocasionando con menos frecuencia crisis adrenal aguda. El daño subagudo de muchas entidades asociadas rara vez induce una crisis, mientras la destrucción de la hipófisis o las suprarrenales o la interrupción aguda de la terapia esteroide siguen siendo las causas más frecuentes de crisis adrenales (Tablas 2 y 3).

Además de la presentación clínica de la insuficiencia adrenal crónica y la forma aguda, ha causado interés la insuficiencia adrenal relativa (IAR) en los pacientes críticamente enfermos. En ellos la secreción de cortisol y de CRH por lo menos en la primeras fases de la enfermedad se mantiene, pero su concentración no es adecuada para la situación de estrés.

\section{Epidemiología}

La incidencia de ISA en pacientes de cuidado intensivo es difícil de estimar ya que la población es diversa y en los estudios se han usado diferentes criterios diagnósticos. La incidencia en la población general no supera el $0,1 \%$. Aunque la ISA completa es rara en enfermos críticos, existen muchos reportes respecto a la insuficiencia relativa o parcial como causa de morbilidad asociada.

Las tasas de incidencia oscilan en rangos muy amplios que van desde $0 \%$ hasta $77 \%$, siendo aceptado entre 10 y $30 \%$. Existen situaciones clínicas como el cho- 


\section{Tabla 2. Causas de insuficiencia adrenal primaria}

I. Enfermedad autoinmune (única o en la insuficiencia pluriglandular).

2. Infecciones adrenales (TBC, VIH, infecciones por hongos).

3. Metástasis bilaterales.

4. Adrenelectomía

5. Deficiencia de enzimas adrenales.

6. Hemorragia adrenal o necrosis causadas por sepsis o coagulopatía.

7. Idiopática.

8. Inducida por fármacos.

9. Adrenoleucodistrofia.

que séptico que en las diferentes series se encuentra reportado hasta el 50\%. Las tasas de mortalidad se incrementarían en aquellos pacientes con insuficiencia relativa. La evidencia sobre el aumento de la mortalidad del $22 \%$ al $44 \%$ con el ingreso del etomidato (inhibidor de la 11-beta hidroxilasa, enzima crítica en el paso final de cortisol) apoyaría estas cifras. Es de particular interés el crecimiento y la detección de ISA en algunos grupos como los pacientes infectados por VIH que hasta el $20 \%$ podría desarrollarla.

\section{Presentación clínica de la ISA}

Las manifestaciones de la enfermedad primaria son de característica insidiosa con síntomas no específicos, fatiga crónica, debilidad y letargia, anorexia y pérdida de peso, hipotensión postural, distensión abdominal y pérdida de la libido, incluyendo caída del vello púbico y axilar en las mujeres. La hiperpigmentación se encuentra presente en las zonas no expuestas al sol, siendo este rasgo parte de la sospecha clínica. Las anormalidades de los electrolitos con hiponatremia, hipercalemia y en ocasiones hipercalcemia con hipoglicemia, apoyan el diagnóstico.
Tabla 3. Causas de insuficiencia adrenal secundaria

I. Tumor hipotalámico o hipofisiario.

2. Irradiación hipofisiaria.

3. Cirugía hipofisiaria.

4. Trauma cerebral o hipofisiario.

5. Infecciones o inflamación de origen autoinmune en la región hipofisiaria (sarcoidosis, hipofisitis).

6. Necrosis de la hipófisis o sangrado (síndrome de Sheehan).

7. Interrupción abrupta de terapia esteroide prolongada.

8. Causas de ISA con incremento del riesgo para crisis adrenal.

La crisis adrenal es atribuible a la deficiencia de mineralocorticoides, por lo tanto la presentación predominante es la hipotensión o el choque, este último asociado con una disminución del sodio y del volumen intravascular, pero con exceso de prostaglandinas (prostaciclina) y disminución de la respuesta a la norepinefrina y la angiotensina II, que favorecen el colapso circulatorio.

\section{Disfunción suprarrenal en el paciente crítico}

La falla en la secreción de las glándulas suprarrenales fue el primer factor implicado en la fisiopatología del choque circulatorio asociado con sepsis. Los datos más recientes del rol de la actividad normal de las suprarrenales en la respuesta inmediata al estrés considerando como ISA los niveles séricos de cortisol menores de $100 \mathrm{nmol} / \mathrm{L}$; el concepto de IAR se presenta cuando a pesar de los niveles normales o altos de cortisol en los pacientes críticos, se consideran inadecuados para el nivel de estrés y sin capacidad de responder a agresiones mayores, demostrado por la falta de respuesta a la aplicación de ACTH exógena. 
El incremento de las concentraciones de cortisol en el paciente crítico refleja un balance entre el aumento de la tasa de secreción y la reducción de la degradación hepática. En aquellos sépticos con enfermedad hepática se observan mayores niveles de cortisol que cuando no hay este trastorno. Dichas situaciones evidencian un amplio rango de niveles de cortisol desde muy bajos hasta concentraciones extremadamente elevadas, favorecidos por una alta respuesta a la ACTH por parte de la corteza adrenal. La respuesta a la ACTH exógena también se ha postulado como prueba para documentar la función inadecuada en diferentes rangos, con $500 \mathrm{nmol} / \mathrm{L}$ como la respuesta máxima al estímulo y menores de 250 ó $200 \mathrm{nmol} / \mathrm{L}$ a los 30 a 60 minutos luego del estímulo se consideran como ISA. Estos pacientes con respuesta inadecuada tienen mayor mortalidad que los que responden en forma apropiada, aunque esta afirmación no está soportada en todos los estudios clínicos disponibles.

El cortisol plasmático por lo regular está elevado, lo que cursa con un aumento en la mortalidad, reflejando la severidad de la enfermedad. La ISA se ha documentado en infecciones severas como la meningococcemia e infecciones por gram negativos, con hallazgos patológicos sugestivos de necrosis hemorrágica adrenal, asociándose con mayor mortalidad cuando no se inicia el tratamiento adecuado.

La sepsis puede afectar la función adrenocortical por varios mecanismos: disminución de la unión de la CRH con su receptor de membrana, inhibición de la producción de AMPc y la conversión de colesterol a pregnenolona. El factor de crecimiento beta inhibe las acciones de ACTH sobre las células de la corteza adrenal.

Las corticostatinas, una familia de péptidos originados en diversos tipos de células incluyendo los granulocitos, compiten con los sitios de unión de la ACTH en las células adrenales y pueden inhibir la esteroidogénesis. La respuesta inapropiada al estrés puede agravar la resistencia tisular a los GC (Tabla 4).
Tabla 4. Mecanismos de resistencia periférica a GC

I. Reducción del acceso de cortisol a sitios de inflamación por la disminución de globulina transportadora circulante.

2. Modulación del nivel local de cortisol por una reducción del complejo cortisol-globulina.

3. Reducción del número y afinidad de los receptores de GC, expresados sobre los linfocitos.

4. Incremento de la conversión de cortisol en cortisona inactiva por el aumento de la actividad de la I I-B-hidroxideshidrogenasa estimulada por IL-2, IL-4 e IL-I3.

La presentación clínica de la IAR con choque hiperdinámico dependiente de catecolaminas que responde a esteroides a pesar de la ausencia de ISA, podría explicarse por la desensibilizacion en la respuesta celular a los GC. Estos son necesarios para el adecuado acople de los receptores adrenérgicos y las altas concentraciones de catecolaminas en el paciente crítico generarían una regulación negativa de los receptores. Las altas dosis de GC reacoplarían los receptores y mejoraría la respuesta del tono vascular. En aquellos con requerimientos prolongados de ventilación mecánica junto con hipotermia, hipercalemia e hiponatremia también debe sospecharse esta entidad clínica.

\section{Diagnóstico de la ISA}

Muchas pruebas se han propuesto para el diagnóstico de ISA en cuidado intensivo en razón del amplio espectro de los signos y síntomas, siendo el nivel de cortisol sérico la primera elección.

En situaciones no agudas se miden los niveles de cortisol matutino, cuando son elevados excluyen el diagnóstico (niveles superiores a $20 \mathrm{ug} / \mathrm{dL}$ ), pero en el paciente crítico pueden estar altos en cualquier momento del día. Hay que tener en cuenta que la unión del cortisol con su proteína transportadora puede estar afectado (aunque raro) en desórdenes genéticos, por 
Tabla 5. Diagnóstico de la insuficiencia adrenal crónica

\section{Prueba de selección:}

- Cortisol plasmático libre en la mañana' y CRH plasmática.

- Aldosterona plasmática y actividad de renina.

- Excreción urinaria de cortisol libre.

\section{Prueba de estimulación:}

A. Estimulación de la función adrenal (sospecha de insuficiencia primaria) (I a 250 ug* de CRH) midiendo el cortisol a los 30 y 60 minutos. $^{2}$

B. Estimulación del eje hipófosis-adrenal por hipoglicemia inducida por insulina (sospecha de insuficiencia de origen hipotálamo-hipófosis).

- Aplicar insulina regular 0.1 unidades IV.

- Medir el cortisol y $\mathrm{CRH}^{3}$ a los 30-60 y 90 minutos luego de iniciada.

' Niveles de cortisol normal: de 10 a 20 ug/dL entre las 6 y 8 a.m. si la proteina fijadora es normal.Valores menores a $3 \mathrm{ug} / \mathrm{dL}(80 \mathrm{nmol} / \mathrm{L})$ son muy sugestivos de ISA y entre 3 y $10 \mathrm{ug} / \mathrm{dL}$ generan la sospecha.

${ }^{2}$ Niveles de cortisol mayores de $20 \mathrm{ug} / \mathrm{dL}$ (con proteina fijadora presumiblemente normal) excluyen una causa primaria pero no secundaria.

* Las dosis de I ug y 250 ug de $\mathrm{CRH}$ son equivalentes.

${ }^{3}$ El nivel de cortisol en hipoglicemia casi siempre excede $20 \mathrm{ug} / \mathrm{dL}$ ( $550 \mathrm{nmol} / \mathrm{L}$. Al mismo tiempo los niveles de $\mathrm{CRH}$ ayudan a definir el origen (hipotálamohipófisis o adrenal). la exposición a estrógenos o enfermedad hepática, renal o bien una afección aguda, pues el nivel total no refleja el cortisol libre. La CRH basal se encuentra aumentada en casos de ISA primaria $(>100 \mathrm{pg} / \mathrm{mL})$ $\mathrm{y}$ en hipotiroidismo primario. También se detectan altos niveles de renina y bajos de aldosterona en la ISA primaria. La prueba dinámica con la aplicación de CRH o la hipoglicemia inducida por insulina a menudo se utiliza para realizar el diagnóstico de ISA (Tabla 5), pero es necesario explorar la etiología de la enfermedad (Tablas 2 y 3 ).

En el paciente crítico el diagnóstico de la ISA radica en que debe instaurarse la intervención terapéutica temprana. El primer rasgo clínico que hace sospechar la misma es la hipotensión no explicada, sobre todo en pacientes de alto riesgo como ocurre en aquellos con VIH, cuando reciben corticoides, cursan con enfermedad autoinmune conocida o hay historia de fatiga crónica e hiperpigmentación, o bien quienes debutan con laboratorios sugestivos de crisis adrenal aguda (Tabla 6).

El perfil hemodinámico de estos pacientes por lo regular evidencia un alto gasto cardíaco con baja resistencia periférica. Aunque los síntomas no sean específicos de ISA la inestabilidad hemodinámica, la dependencia de vasopresores a pesar del control de la infección y la ocurrencia de hipoglicemia e hipereosinofilia hace sospechar el diagnóstico.

\section{Prueba de estimulación con ACTH}

La estimulación rápida con $\mathrm{ACTH}$ se ha usado para detectar la hiporrespuesta adrenocortical por su sencillez (Tabla 6). Consiste en medir el cortisol a los 30 y 60 minutos luego de la aplicación en bolo de 250 ug de CRH. Existen muchos niveles descritos en la literatura pero los menores de $15 \mathrm{ug} / \mathrm{dL}$ (414 nmol) parecen identificar mejor a los pacientes que junto con rasgos clínicos y paraclínicos de ISA, se beneficiarían del tratamiento con reemplazo de cortisol. En contraste con los pacientes no críticos, el incremento en la respuesta luego de la administración de CRH tiene implicaciones pronósticas. 
Tabla 6. Procedimientos diagnósticos en la crisis adrenal aguda

\section{Laboratorios:}

- Sodio, potasio y bicarbonato.

- Cortisol plasmático.

- CRH plasmática, renina y aldosterona.

\section{Test de estimulación con CRH:}

Aplicar 250 ug de $\mathrm{CRH}$ midiendo el cortisol a los 30 minutos.

La crisis adrenal no es sugestiva si:

- El cortisol basal es mayor de 20 ug/dL.

- El cortisol post CRH es mayor de 20 ug/dL.

Pequeños incrementos, menores de $9 \mathrm{ug} / \mathrm{dL}$ (250 nmol/L), del cortisol basal a niveles muy altos se asocian con mayor mortalidad. Valores menores de $15 \mathrm{ug} / \mathrm{dL}$ junto con una pobre respuesta al estímulo con $\mathrm{CRH}$ indicarían la posibilidad de ISA e inicio de terapia.

Es importante anotar que los pacientes con incrementos de cortisol mayores de $9 \mathrm{ug} / \mathrm{dL}$ y altos niveles basales ( $>34 \mathrm{ug} / \mathrm{dL}$ ) asociados con características clínicas sugestivas de ISA, hacen pensar en resistencia periférica a los corticoides. No está definido si es necesario realizar todas las pruebas descritas, pero si durante el desarrollo de la enfermedad se presentan signos y síntomas que sugieran ISA o deterioro de la condición clínica, deben practicarse.

Las dosis de CRH se han estudiado en diversas series clínicas encontrando que las de 1 ug son más sensibles para el diagnóstico que las de 250 ug. Siraux et al. describieron un grupo de pacientes respondedores a dosis altas durante la prueba pero no a bajas dosis, asociándolos con pobres resultados clínicos. Además durante la sepsis algunas citoquinas secretadas por el tejido adiposo inducen resistencia a la ACTH y las bajas dosis de ACTH podrían ser insuficientes para estimular la respuesta de la corteza adrenal.

A este respecto muchos estudios describen falsos negativos con altas dosis de CRH en pacientes con ISA secundaria. La atrofia adrenal o el daño funcional luego de la supresión con ACTH requiere semanas para desarrollarse y se presenta en diferentes grados de severidad en pacientes con enfermedades hipofisiarias o en previo tratamiento con esteroides. La dosis de $250 \mathrm{ug}$ incrementa la concentración de ACTH a $60.000 \mathrm{pg} / \mathrm{mL}$ lo cual excede los $100 \mathrm{pg} /$ $\mathrm{mL}$ necesarios para estimular la corteza adrenal. Este hecho obviamente alteraría los resultados en pacientes con ISA, por esta razón se recomienda realizar adicionalmente la prueba de hipoglicemia inducida por insulina o aquella con metirapona. La última no se recomienda en pacientes críticos porque podría desencadenar una crisis adrenal. Estas dos pruebas dinámicas evalúan el eje hipotálamohipófisis-adrenal, pero son dispendiosas.

\section{Tratamiento de la ISA}

El tratamiento de la crisis en la enfermedad de Addison está dirigido al reemplazo electrolítico, de líquidos y uso de hidrocortisona; hay que iniciarlo en forma oportuna y acompañarlo de la toma previa de los laboratorios (Figura 1). Cuando se confirma la ISA o es muy sugestiva por la clínica y los laboratorios, se debe iniciar la reposición con hidrocortisona en dosis entre $150 \mathrm{mg}$ y $300 \mathrm{mg}$ por vía parenteral por dos a tres días.

Los beneficios observados en la menor mortalidad de los pacientes con choque séptico que recibieron esteroides por más de cinco días y dosis menores de $300 \mathrm{mg}$ fueron evidentes. Sin embargo, existen otros trabajos que utilizaron reposición con esteroides, sin demostrar que dosis bajas de hidrocortisona en la crisis adrenal mejoren la supervivencia en estos pacientes. Los efectos observados fueron la disminución del soporte vasopresor a los 7 y 28 días, reduciendo la severidad de la inflamación, la intensidad y duración de la falla orgánica. 


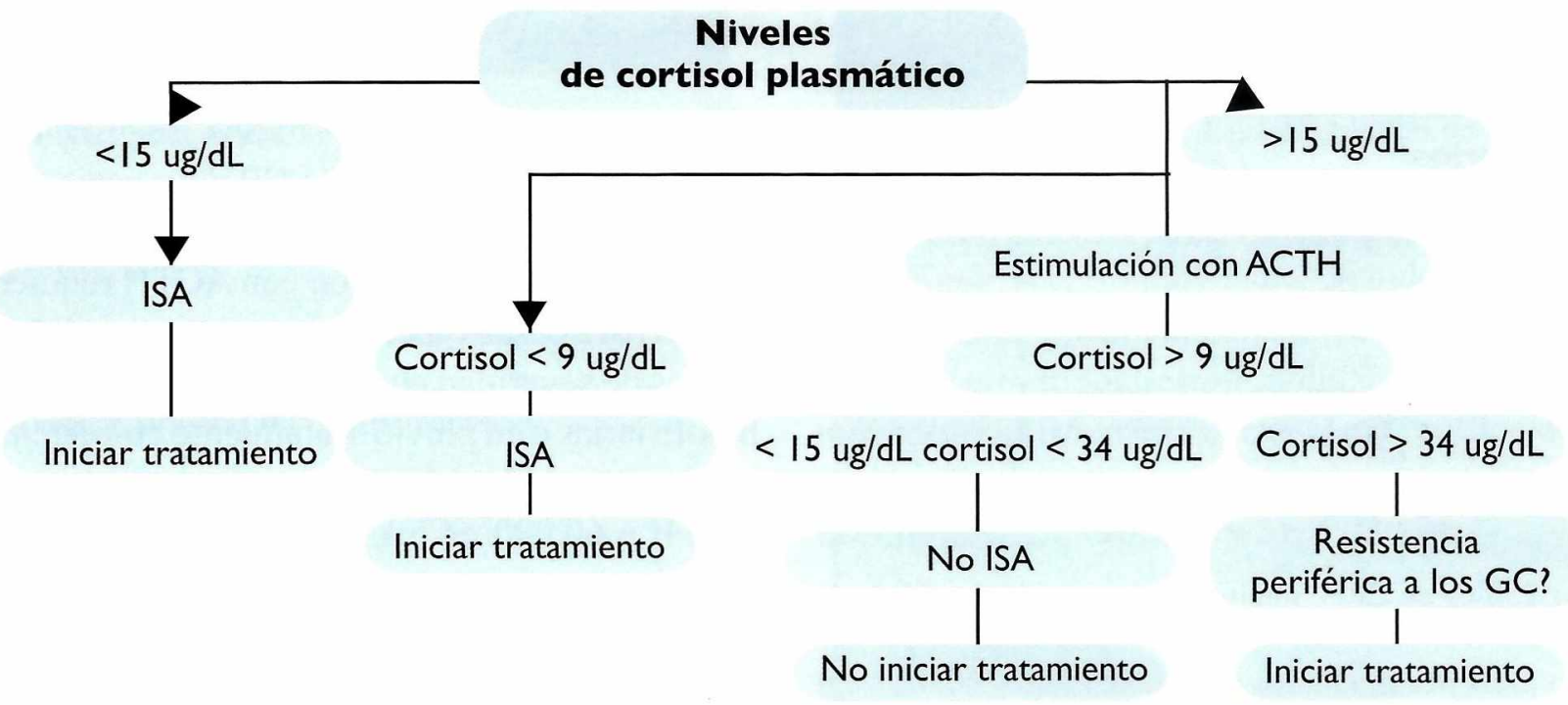

Figura I. Algoritmo diagnóstico de la ISA.

Sospecha clínica de ISA

\section{Prueba de estimulación con CRH}

Cortisol sérico $>15 \mathrm{ug} / \mathrm{dL}$

Postestímulo $>20 \mathrm{ug} / \mathrm{dL}$

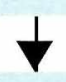

Considerar el uso de GC por I a 2 días si no hay respuesta a los vasopresores con LEV.
Cortisol sérico $<15 \mathrm{ug} / \mathrm{dL}$ Postestímulo $<20$ ug/dL

Iniciar esteroides a dosis de estrés (hidrocortisona 150 a $200 \mathrm{mg} /$ día) y mantener por 2 a 3 días luego de que los parámetros hemodinámicos mejoren.

Reevaluar la necesidad de continuar esteroides si no hay un significativo cambio luego de 2 a 3 días.

Figura 2. Algoritmo terapéutico en insuficiencia adrenal.

No existe evidencia concreta sobre el reemplazo mineralocorticoide ya que los resultados de la terapia con cortisol sobre el sangrado gastrointestinal, sobreinfección o hiperglicemia no se han descrito. El uso de fludrocortisona ha sido seguro en pacientes con choque séptico dependiente de vasopresores y se ha asociado con una reducción de la mortalidad a corto y mediano plazo. El efecto aditivo de la fludrocortisona a la hidrocortisona aun falta por aclararse y no hay datos conclusivos sobre la modalidad de tratamiento y la suspensión del mismo. El medicamento de elección continúa siendo la hidrocortisona, en dosis menores de $300 \mathrm{mg}$ al día, aunque la administración en infusión continua o en bolos no se ha estudiado. 
Una alternativa para iniciar el tratamiento sin interferir con la toma de cortisol y la estimulación con ACTH es el uso de dexametasona entre 4 y $6 \mathrm{mg}$ cada 12 horas, o utilizar $4 \mathrm{mg}$ de dexametasona seguido de una prueba rápida con ACTH y luego iniciar el reemplazo con hidrocortisona (Figura 2). La prednisona y la cortisona no se utilizan en los pacientes con hipotensión porque requieren hidroxilación para crear el principio activo. El reemplazo de dehidroepiandosterona en las mujeres con ISA primaria debe iniciarse pronto ya que en múltiples estudios clínicos se han demostrado los efectos benéficos de su uso.

\section{Lecturas recomendadas}

- Arafah BM. Hypothalamic pituitary adrenal function during critical illness: limitations of current assessment methods. J Clin Endocrinol Metab. 2006 Oct;91(10):372545. Epub 2006 Aug 1.

- Bouillon R. Acute Adrenal Insufficiency. Endocrinol Metab Clin North Am. 2006 Dec;35(4):767-75.

- Cooper MS, Stewart PM. Current Concepts: Corticosteroid Insufficiency in Acutely Ill Patients. N Engl J Med 2003;Feb 20; 348(8):727-34.

- De Jong MF, Beishuizen A, Spijkstra JJ, Groeneveld AB. Relative adrenal insufficiency as a predictor of disease severity, mortality, and beneficial effects of corticosteroid treatment in septic shock. Crit Care Med. 2007 Aug;35(8):1896-903.

- Gonzalez H, Nardi O, Annane D. Relative adrenal failure in the ICU: an identifiable problem requiring treatment. Crit Care Clin. 2006 Jan;22(1):105-18.

- Hamrahian A. Adrenal function in critically ill patients: how to test? When to treat? Cleve Clin J Med. 2005 May;72(5):427-32.

- Lukert BP. Editorial: glucocorticoid replacement-how much is enough? J Clin Endocrinol Metab. 2006 Mar;91(3):793-4.

- Morris J. et al. Adrenal Insufficiency, Hear tRate Variability, and Complex Biologic Systems: A Study of 1,871 Critically III Trauma Patients. J Am Coll Surg Vol.204, No.5, May 2007: 885-892.

- Raghavan M, Marik PE. Stress hyperglycemia and adrenal insufficiency in the critically ill. Semin Respir Crit Care Med. 2006 Jun;27(3):274-85.

- Salvatori R. Adrenal insufficiency. JAMA. 2005 Nov 16;294(19):2481-8.

- Shenker Y, Skatrud JB. Adrenal insufficiency in critically ill patients. Am J Respir Crit Care Med. 2001 Jun;163(7):1520-3.

- Stewart PM, Quinkler MO. Mineralocorticoid de.ciency. In: Degroot LJ, Jameson JL, editors. Endocrinology. Philadelphia: WB Saunders; 2005. p. 2491-9.

- Torrey SP. Recognition and management of adrenal emergencies. Emerg Med Clin North Am. 2005 Aug;23(3):687-702.
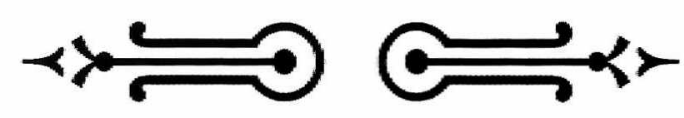